\title{
Altered fatty acid composition of Nicotiana benthamiana and Nicotiana excelsior leaves under transient overexpression of the cyanobacterial desC gene
}

\author{
M. BERESTOVOY*, O.S. PAVLENKO, A.A. TYURIN, E.N. GORSHKOVA, \\ and I.V. GOLDENKOVA-PAVLOVA
}

Timiryazev Institute of Plant Physiology, Russian Academy of Sciences, Moscow 127276, Russia

\begin{abstract}
Transient heterologous gene expression in two model plant species, Nicotiana benthamiana and N. excelsior, has been used to study the localization of the heterologous $\Delta 9$ acyl-lipid desaturase ( $\Delta 9$ desaturase) of Synechococcus vulcanus in different cell compartments and its functional activity in the cases of the cytosol, chloroplast, and endoplasmic reticulum (ER) localization. The functional activity and substrate specificity of the heterologous desaturase under the conditions of transient expression have been confirmed by comparison of fatty acid (FA) profiles. The $\Delta 9$ desaturase, responsible for the synthesis of oleic and palmitoleic acids, has also been shown to strongly promote the accumulation of polyunsaturated FAs. The results convincingly demonstrate that the $\Delta 9$ desaturase of the thermophilic cyanobacterium transiently expressed in two Nicotiana species considerably alters lipid metabolism in their leaves towards a higher FA unsaturation. The functional activity of $\Delta 9$ desaturase depends on both the model plant species, $N$. benthamiana or $N$. excelsior, and the cellular localization of the enzyme. The method of transient expression of heterologous genes in plants is highly effective, inexpensive, and not time-consuming, which makes it attractive for estimating the functional activity and/or substrate specificity of heterologous desaturases.
\end{abstract}

Additional key words: cellular compartments, $\Delta 9$ desaturase, oleic and palmitoleic acids, signal sequences.

\section{Introduction}

Fatty acids (FAs) and their derivatives constitute energy stores of plant seeds and play the key role in plant metabolism. They are the main structural components of membranes and can serve as the precursors of signal molecules involved in response to stress factors and during plant development (Dar et al. 2017).

It is currently believed that lipids are synthesized via the prokaryotic and eukaryotic pathways in plastids and endoplasmic reticulum (ER), respectively. Most plant FA desaturases involved in the formation of unsaturated FAs are also located in these cell compartments (Los et al. 2013, Wang et al. 2016, Dar et al. 2017). The eukaryotic and prokaryotic pathways interact with each other through a reversible exchange of FAs between the plastids and ER, in particular, in the case of sequential desaturation of FAs by desaturases.
Desaturases catalyze the transformation of single bonds between carbon atoms $(\mathrm{C}-\mathrm{C})$ in the acyl chains of FAs into double bonds $(\mathrm{C}=\mathrm{C})$ (Los et al. 2013), thereby converting saturated FAs into unsaturated ones. Solvable acyl-[acyl-carrier-protein] desaturases and membranebound acyl-lipid desaturases are responsible for FA desaturation in plant cells (Los et al. 2013). The $\Delta 9$ acyl[acyl-carrier-protein] desaturase ( $\Delta 9$ desaturase) plays the key role in the formation of unsaturated FAs necessary for maintaining the optimal liquid-crystal structure of plant cell membranes because it forms the first double bond in the FA chain (López Alonso et al. 2003). For example, $\Delta 9$ desaturase converts stearic acid (18:0) into oleic acid (18:1) by introducing a double bond between the ninth and tenth carbon atoms in the FA acyl chain (TroncosoPonce et al. 2016), thereby providing the substrate for other desaturases sequentially forming the second (position $\Delta 12$ ), third $(\Delta 15)$, and subsequent double bonds

Submitted 2 August 2019, last revision 20 October 2019, accepted 21 November 2019

Abbreviations: DBI - double bond index; ER - endoplasmic reticulum; FA - fatty acid; FAME - fatty acid methyl ester; GC-MS - gas chromatography-mass spectrometry; GFP - green fluorescent protein; PDR - fatty acid C16:1 to C16:0 ratio; PUFA - polyunsaturated fatty acid; RuBisCO - ribulose-1,5-bisphosphate carboxylase/oxygenase; SDR - fatty acid C18:1 to C18:0 ratio; TLC - thin layer chromatography.

Acknowledgements: We are grateful to Dr. Roman Sidorov (K.A. Timiryazev Institute of Plant Physiology, the Russian Academy of Sciences) for his help in analyzing the fatty acid composition.

* Corresponding authors; e-mail: m.berestovoy1181@gmail.com 
(Napier 2007, Los et al. 2013, Wang et al. 2016, Dar et al. 2017). Thus, the desaturation of 18:0 to $18: 1$ catalyzed by $\Delta 9$ desaturase is one of the crucial stages controlling the content of unsaturated FAs in the cells.

Gene engineering approaches, such as constitutive expression of heterologous desaturase genes (Peng et al. 2018, Shi et al. 2018) and the RNA interference technology as a means to inhibit the expression of the target desaturase gene (Wang et al. 2010), are often used as convenient models to study the role of desaturases, including $\Delta 9$ desaturase, in plant physiology. Specifically, it has been convincingly demonstrated that constitutive expression of heterologous $\Delta 9$ desaturase genes in transgenic plants leads to effective conversion of stearic acid (18:0) into oleic acid (18:1), which is accompanied by considerable changes in the unsaturation of FAs, including polyunsaturated FAs (PUFAs) in membrane lipids (Orlova et al. 2003, Craig et al. 2008, Gerasymenko et al. 2015, Zhang et al. 2015).It is currently assumed that plant enzymes of this group are characterized by diverse lipid substrates, region-specific desaturation, and the capacity for functioning in different cell compartments, which deserves further study.

It should be noted that gene engineering approaches, which usually entail obtaining transgenic plants, are expensive and time-consuming. In this connection, researchers studying the functional activity, substrate specificity, and physiological role of the protein products of target genes switch from obtaining stable plant transformants to using systems of transient expression of heterologous genes in model plants, such as $N$. benthamiana and $N$. excelsior (Anwar et al. 2018, Ji et al. 2018, Sheludko et al. 2018, Thodberg et al. 2018, Gerasymenko et al. 2019, Li et al. 2019).

Here, we used transient expression of the $\operatorname{des} \mathrm{C}$ gene from Synechococcus vulcanus, which encodes $\Delta 9$ desaturase, in two model plants, $N$. benthamiana and $N$. excelsior, to estimate the applicability of this method to studying the functional activity of desaturases, including the cases when the heterologous $\Delta 9$ desaturase was located in different cell compartments, namely, the cytosol, chloroplasts, and endoplasmic reticulum (ER).

\section{Materials and methods}

Engineering of plant expression vectors: Standard molecular cloning procedures and PCR protocols were used. Restriction endonucleases, T4 DNA ligase, Taq and Pfu DNA polymerases, and phosphatases were used according to the manufacturers' protocols (Promega, Madison, USA; Fermentas, Vilnius, Lithuania).

The expression vectors were obtained in several steps. First, the nucleotide sequence of the desC gene encoding the $\Delta 9$ acyl-lipid desaturase of Synechocystis vulcanus was obtained by means of PCR using the pQEdesC plasmid (Maali et al. 2007) as a template, the GGGCCCACATCATCCTTAGAA sequence as a forward primer (F1), and the GAATTCGGACAACGCTTTGGG sequence as a reverse primer (R1), with the ApaI and $E c o R I$ restriction sites introduced into the forward and reverse primers, respectively. The PCR product was cloned into the pPGG vector (Vyacheslavova et al. 2012) preliminarily hydrolyzed at the ApaI and EcoRI sites to obtain the pPGG-D9 vector. The desC-egfp hybrid gene was constructed in the following steps. Firstly, the nucleotide sequence of the egfp gene encoding green fluorescent protein (GFP) was obtained by means of PCR using the pQE-egfp plasmid as a template, the GAATTCGTGAGCAAGGGCGAG sequence as a forward primer(F2), and the CCCGGGCTTGTAGTACAGCTCGT sequence as a reverse primer (R2), with the EcoRI and SmaI restriction sites introduced into the forward and reverse primers, respectively (Piruzian et al. 2002). The resultant PCR fragment was cloned into the pPGG-D9 plasmid preliminarily hydrolyzed at the EcoRI and SmaI sites to obtain the pPGG-D9E plasmid. The correctness of the hybrid gene construction was confirmed by sequencing.

The pVIG-D9 plant expression vector carrying the des $\mathrm{C}$ gene was obtained by cloning the SacI-XbaI fragment of the pPGG-D9 plasmid into the pVIG-T 1A vector (Vyacheslavova et al. 2012) preliminarily hydrolyzed at the ApaI and SmaI sites. The pVIG-D9E plant expression vector carrying the desC-egfp hybrid gene was obtained by cloning the ApaI-SmaI fragment of the pPGG-D9E plasmid into the pVIG-T 1A vector (Vyacheslavova et al. 2012) preliminarily hydrolyzed at the ApaI and SmaI sites.

The plant expression vector carrying the des $\mathrm{C}$ gene fused with the sequence for the transit peptide of the small chain of RuBisCO (Lch) of Arabidopsis thaliana (gene ats $1 A, N C B I, \mathrm{X} 13611$ ) was obtained in several steps. At the first step, the Lch sequence encoding the transit peptide of the RuBisCO small chain was obtained by means of PCR using the pVIG-S2B plasmid (Vyacheslavova et al. 2012) as a template, the GGTACCATGGCTTCTATGATATC sequence as a forward primer (F3), and the GGGCCCCTGATATTCAACTATAT sequence as a reverse primer (R3), with the KpnI and ApaI restriction sites introduced into the forward and reverse primers, respectively. At the second step, the PCR product was cloned into the pVIG-D9 vector preliminarily hydrolyzed at the KpnI and ApaI sites to obtain the pVIG-Lch-D9

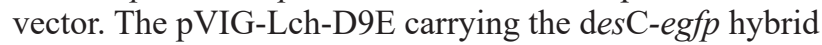
gene fused with the sequence for the transit peptide of the small chain of RuBisCO (Lch) was obtained by cloning the ApaI-SmaI fragment of the pVIG-D9E plasmid into the pVIG-Lch-D9 vector.

The plant expression vector carrying the des $\mathrm{C}$ gene fused with the sequences ensuring protein transport in the endoplasmic reticulum (ER) was obtained as follows. First, the LeB4 sequence encoding the transport protein was obtained by means of PCR using the pVIG-T 2D plasmid (Vyacheslavova et al. 2012) as a template, the GGTACCATGTCCAAACCTTTTCT sequence as a forward primer (F4), and the GGGCCCTGCTAAACATGTGCT sequence as a reverse primer (R4), with the KpnI and ApaI sequences introduced into the forward and reverse primers, respectively. The PCR product was cloned into the $\mathrm{pVIG}-\mathrm{D} 9$ vector preliminarily hydrolyzed at the KpnI and ApaI sites to obtain the pVIG-LeB4-D vector. Then, the SmaI-XbaI fragment of 
the pVIG-T 2D plasmid carrying the sequence encoding the signal of protein retention in the ER (SRKDEL) and the polyadenylation signal was cloned into the pVIGLeB4-D9 vector preliminarily hydrolyzed at the SmaI and XbaI sites to obtain the pVIG-D9-ER vector. The pVIGD9E-ER vector carrying the $\operatorname{desC}$-egfp hybrid gene fused with the $L e B 4$ sequence encoding the transport peptide and the sequence encoding SRKDEL was obtained by cloning the ApaI-SmaI fragment of the pVIG-D9E plasmid into the $\mathrm{pVIG-D9-ER} \mathrm{vector.}$

The $p$ VIG-E expression vector carrying the egfp gene of GFP obtained earlier was used as a control (Vyacheslavova et al. 2012).

The correctness of the fusion of the genes with the corresponding leader signals in the plant expression vectors pVIG-Lch-D9, pVIG-Lch-D9E, pVIG-D9-ER, and pVIG-D9E-ER was confirmed by sequencing.

Plant species, Agrobacterium strains, and agroinfiltration: Nicotiana benthamiana Domin and Nicotiana excelsior J.M. Black were grown hydroponically at a temperature of $22 \pm 2{ }^{\circ} \mathrm{C}$, a 8 -h photoperiod, an irradiance of $100 \mu \mathrm{mol} \mathrm{m}{ }^{-2} \mathrm{~s}^{-1}$, and a relative humidity of ?? \%. Knop's solution was used as a nutrient medium. The pVIG-E, pVIG-D9, pVIG-D9E, pVIG-Lch-D9, pVIG-Lch-D9E, pVIG-D9-ER, and pVIG-D9E-ER vectors were used to transform the Agrobacterium tumefaciens GV3101 strain as described earlier (Berestovoy et al. 2018). Transformed bacteria were selected on a medium containing kanamycin and designated according to the expression vector used for the transformation. The transformed agrobacteria were used for agroinfiltration into the abaxial epidermis of the leaves of six-week-old $N$. benthamiana and $N$. excelsior plants by means of a syringe without a needle as described earlier (Berestovoy et al. 2018). After the agroinfiltration, the plants were cultured under the same conditions for seven days. Agroinfiltrated leaf fragments were used in subsequent analysis.

Protoplast isolation and fluorescence microscopy: Protoplasts were isolated from the leaf fragments with fluorescent areas as described earlier (Tyurin et al. 2017, Berestovoy et al. 2018). The fluorescence in protoplasts was recorded using an Axio Imager Z2 (Zeiss, Oberkochen, Germany) microscope equipped with an AxioCam MR digital camera and filters No. 38 ( $\lambda_{\text {ex }}$ BP 470 $\mathrm{nm} / 40 \mathrm{~nm}$ and $\lambda_{\mathrm{em}}$ BP $525 \mathrm{~nm} / 50 \mathrm{~nm}$, where ex stands for fluorescence excitation and em for fluorescence emission) and No. $45\left(\lambda_{\text {ex }}\right.$ BP $560 \mathrm{~nm} / 40 \mathrm{~nm}$ and $\lambda_{\text {em }}$ BP $630 \mathrm{~nm} / 75$ $\mathrm{nm}$ ) for recording the GFP fluorescence and chlorophyll autofluorescence, respectively. The fluorescence of intravital preparations was recorded using an ApoTome module (Zeiss), which reduces defocused fluorescence. The images were manually processed using the AxioVision 4.8 software (Zeiss). About 100 protoplasts were analyzed for each localization of the target protein.

Isolation and analysis of fatty acids from total lipids of N. benthamiana and N. excelsior leaf tissues: Samples of agroinfiltrated leaf areas were chopped up with a scalpel, heated to the boiling point for $30 \mathrm{~min}$ in isopropanol containing $0.001 \%(\mathrm{v} / \mathrm{v})$ of an antioxidant (butylated hydroxytoluen) and $1 \mathrm{mg}$ of heptadecanoic acid as an internal standard (Sidorov et al. 2012), homogenized in a mortar, and boiled for $60 \mathrm{~min}$ in a $8 \%(\mathrm{v} / \mathrm{v})$ isopropanol solution of $\mathrm{KOH}$. Isopropanol was evaporated and replaced with an equal volume of water; unsaponifiable lipids were removed using hexane. The solution was acidified to $\mathrm{pH} 2$, and free FAs were extracted with hexane and converted into fatty acid methyl esters (FAMEs) by boiling in a mixture of $10 \mathrm{~cm}^{3}$ of $\mathrm{CH}_{3} \mathrm{OH}$ and $0.5 \mathrm{~cm}^{3}$ of $\mathrm{CH}_{3} \mathrm{COCl}$ for $1 \mathrm{~h}$. FAMEs were purified by thin-layer chromatography (TLC) on a silica gel-coated plate and separated by gasliquid chromatography (GLC) in a capillary column (Sidorov et al. 2012). The double bond index (DBI), C18:1 to $\mathrm{C} 18: 0$ ratio (SDR), and $\mathrm{C} 16: 1$ to $\mathrm{C} 16: 0$ ratio (PDR) were calculated from the following equations: $\mathrm{DBI}=[(\mathrm{M})+2(\mathrm{D})$ $+3(T)] / 100$, where $M$ is the sum of the mass fractions of monounsaturated FAs, D is the sum of the mass fractions of diunsaturated FAs, and $\mathrm{T}$ is the sum of mass fractions of triunsaturated FAs; SDR $=(\% 18: 1) /(\% 18: 0+\% 18: 1)$, where $\% 18: 0$ is the mass fraction of 18:0 FAs and \%18:1 is the mass fraction of $18: 1 \mathrm{FAs} ; \mathrm{PDR}=(\% 16: 1) /(\% 16: 0+$ $\% 16: 1$ ), where $\% 16: 0$ is the mass fraction of 16:0 FAs and $\% 16: 1$ is the mass fraction of 16:1 FAs.

Statistical analysis: All experiments were performed in at least six independent replicates. The results were statistically treated using the Statistica $v$. 9.0 software (Student's $t$-test was used, with differences considered significant at $P \leq 0.05$ ) and Microsoft Office Excel 2007 software.

\section{Results}

Two series of expression vectors were constructed. In the first series comprising pVIG-D9E, pVIG-Lch-D9E, and pVIG-LeB4-D9E-ER, we used the desC-egfp hybrid gene obtained by fusing the $\operatorname{des} \mathrm{C}$ gene encoding the cyanobacterial $\Delta 9$ desaturase and the $e g f p$ gene, whose protein product is GFP optimized for expression in eukaryotic cells (Fig. 1). Note that the egfp initiation codon was removed from the hybrid gene to avoid alternative translation. In the other series comprising the pVIG-D9, pVIG-Lch-D9, and pVIG-LeB4-D9-ER vectors, only the native cyanobacterial des $\mathrm{C}$ gene was used.

The native $(\operatorname{des} \mathrm{C})$ and hybrid (desC-egfp) genes in the pVIG-Lch-D9 and pVIG-Lch-D9E vectors, respectively, had a well-characterized signal in the 5 ' region ensuring the localization of the target proteins into chloroplasts (Fig. 1) (Gerasymenko et al. 2015). In the pVIG-LeB4-D9-ER and pVIG-LeB4-D9E-ER expression vectors, a sequence corresponding to the LeB4 signal peptide was cloned into the 5 ' regions of the target genes (desC and $\operatorname{des} \mathrm{C}-\mathrm{eg} f \mathrm{p}$ ) for localizing the protein product to the ER, and the fragment encoding the signal of protein retention in the ER was introduced into the 3' region (Fig. 1) (Alanen et al. 2011, Vyacheslavova et al. 2012). In the pVIG-Lch-D9, pVIGLch-D9E, pVIG-D9-ER, and pVIG-D9E-ER vectors, the 


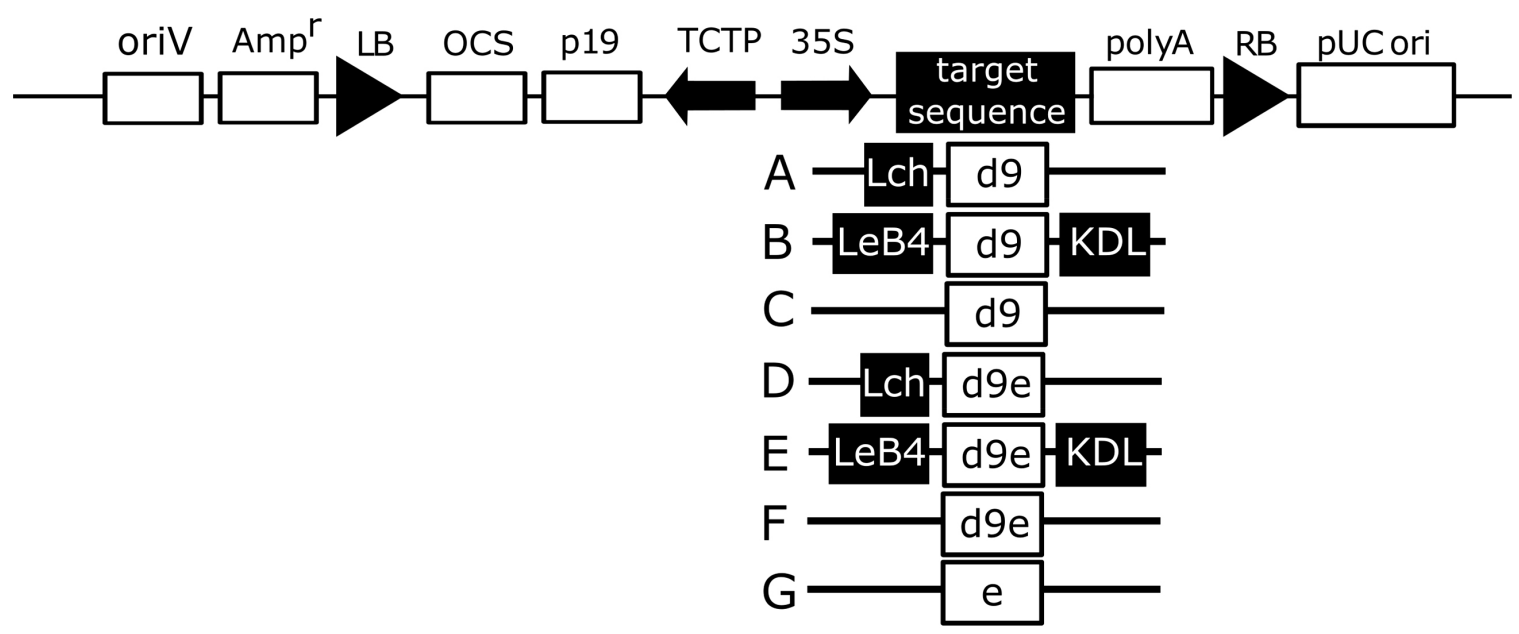

Fig. 1. A schematic representation of plant expression vectors. Each plant expression vector carries the Cymbidium ringspot virus gene $($ CymRSV), which encodes an RNA-silencing suppressor protein p19 under the control of the translationally controlled tumor protein (TCTP) promoter and the termination sequence of the octopine synthase (OCS) gene. oriV - replication start point for Agrobacterium tumefaciens; Ampr - ampicillin resistance cassette; LB and RB - left and right borders, respectively, of the T-DNA region; pUCori origin of replication for Escherichia coli; $35 \mathrm{~S}$ - CaMV 35S promoter; poly-A - polyadenylation signal; Target sequence - the des $\mathrm{C}$ gene sequence or the desC-egfp hybrid gene sequence; Lch - sequence for the transit peptide of $A$. thaliana RuBisCO small subunit (gene ats1A, NCBI, X13611); LeB4 - sequence for the signal peptide for protein localization into endoplasmic reticukum; KDL - endoplasmic reticulum-retention signal; $\mathrm{d} 9$ - des $\mathrm{C}$ gene sequence; $\mathrm{d} 9 \mathrm{e}-\operatorname{des} \mathrm{C}-\mathrm{eg} f p$ hybrid gene sequence; $\varnothing$ - absence of signal peptide for protein localization; e - green fluorescent protein gene sequence $(e g f p)$.

translation-initiation AUG codon was introduced into the 5 ' region of the sequence encoding the Lch and LeB4 signal peptides and removed from the target genes to avoid alternative translation.

In the pVIG-D9 and pVIG-D9E expression vectors, the target genes (desC and $\operatorname{des} \mathrm{C}-\mathrm{eg} f \mathrm{p}$ ) did not contain additional signal sequences in either 5' or 3' region, their expression was controlled only by the $35 \mathrm{~S}$ RNA CaMV promoter, which did not entail any specific compartmentalization of the protein products of these genes (Fig. 1). All the vectors were based on the pVIG-T construct, which we developed earlier specially for effective transient expression of heterologous genes in plants (Vyacheslavova et al. 2012). The T-DNA region of this vector contains the sequence of the gene of the p19 tomato virus protein, a suppressor of posttranscriptional gene silencing under the control of the TCTP (translationally controlled tumor protein) promoter (Fig. 1) (Vyacheslavova et al. 2012). The first series of vectors was used to study the subcellular localization of the heterologous desaturase in plant cells, and the other one, to compare the modification of the FA composition of plant membrane lipids as dependent on the subcellular localization of the heterologous $\Delta 9$ desaturase.

We estimated the localization of the heterologous desaturase with the $\mathrm{C}$-terminal fusion with the fluorescent protein (hereinafter referred to as D9E) in experiments where the signal sequences for target subcellular compartments were used. For this purpose, we used the first series of vectors, the method based on obtaining protoplasts in plant tissue agroinfiltrated with bacterial strains carrying different expression vectors, and fluorescence microscopy.

In the protoplasts obtained after agroinfiltration with the strain carrying the pVIG-Lch-D9E plasmid
(Fig. 1), almost all chloroplasts displayed fluorescence in the yellow spectral region (Fig. 2B). This indicated the chloroplast localization of the D9E fusion protein, which was transferred to these compartments by means of the signal sequence (Lch) of the $A$. thaliana $\mathrm{RuBisCO}$ small chain. The fluorescence signal from D9E was detected in the ER of the protoplasts (Fig. 2C) obtained after agroinfiltration with the strain carrying the pVIG-D9E-ER vector (Fig. 1), where the N-terminal signal peptide LeB4 transferred the D9E protein across the ER membrane, and the C-terminal SRKDEL signal retained D9E in the ER. The protoplasts obtained from the leaves agroinfiltrated with the strain carrying the pVIG-D9E vector (Fig. 1) displayed the fluorescence signal of the D9E fusion protein in the cytosol (Fig. 2A). Thus, the results demonstrated that the signal sequences used ensured the localization of the heterologous $\Delta 9$ desaturase in the target compartments of the plant cell.

In the protoplasts obtained after agroinfiltration with the strain carrying the pVIG-Lch-D9E plasmid (Fig. 1), almost all chloroplasts displayed fluorescence in the yellow spectral region (Fig. 2B, Fig. 1 Suppl.). This indicated the chloroplast localization of the D9E fusion protein, which was transferred to these compartments by means of the signal sequence (Lch) of the $A$. thaliana RuBisCO small chain. The fluorescence signal from D9E was detected in the ER of the protoplasts (Fig. 2C, Fig. 1 Suppl.) obtained after agroinfiltration with the strain carrying the pVIGD9E-ER vector (Fig. 1), where the N-terminal signal peptide LeB4 transferred the D9E protein across the ER membrane, and the C-terminal SRKDEL signal retained D9E in the ER. The protoplasts obtained from the leaves agroinfiltrated with the strain carrying the pVIG-D9E vector (Fig. 1) displayed the fluorescence signal of the D9E 


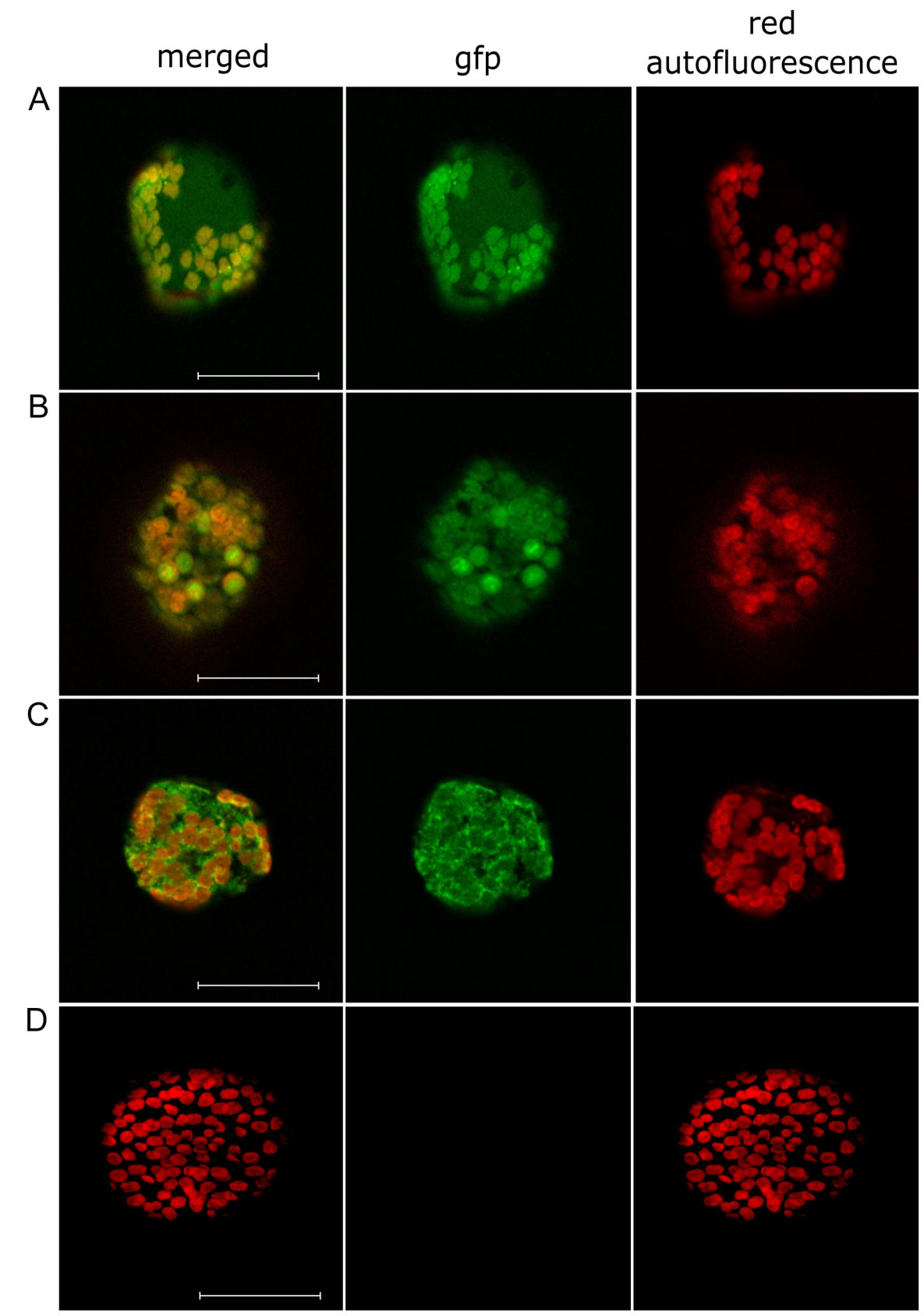

Fig. 2. Subcellular localization of green fluorescent protein (GFP) fusions in Nicotiana benthamiana protoplasts isolated from the cells of the agroinfiltrated leaf area. Transient gene expressions of plant expression vectors, with leader signal sequences providing their localization in various compartments of plant cells, demonstrate that the GFP fusions localized in cytosol $(A)$, chloroplasts $(B)$, endoplasmic reticulum $(C)$, and non-transformed protoplasts $(D)$. The merged images include the GFP channel (green) and the chloroplast autofluorescence channel $(\mathrm{red})$. Each picture represents a single protoplast. All images obtained using the single-sectioned fixed focus plane produced by the ApoTome module (Zeiss). The scale bar is $40 \mu \mathrm{m}$.

fusion protein in the cytosol (Fig. 2A, Fig. 1 Suppl.). Thus, the results demonstrated that the signal sequences used ensured the localization of the heterologous $\Delta 9$ desaturase in the target compartments of the plant cell.

It should be noted that that transient gene expression in plants has not been used before to estimate the functional activity of desaturases. This method entails wounding, if only slight, of the leaves during agroinfiltration. It is known that the expression of plant FA desaturases, including $\Delta 9$ desaturase, may be activated in response to wounding (Dar et al. 2017, Xue et al. 2018). Therefore, we first attempted to determine whether the FA profile was altered after the agroinfiltration and, hence, whether the transient gene expression method could be used to subsequently estimate the functional activity of desaturase.

For this purpose, we compared the FA compositions 


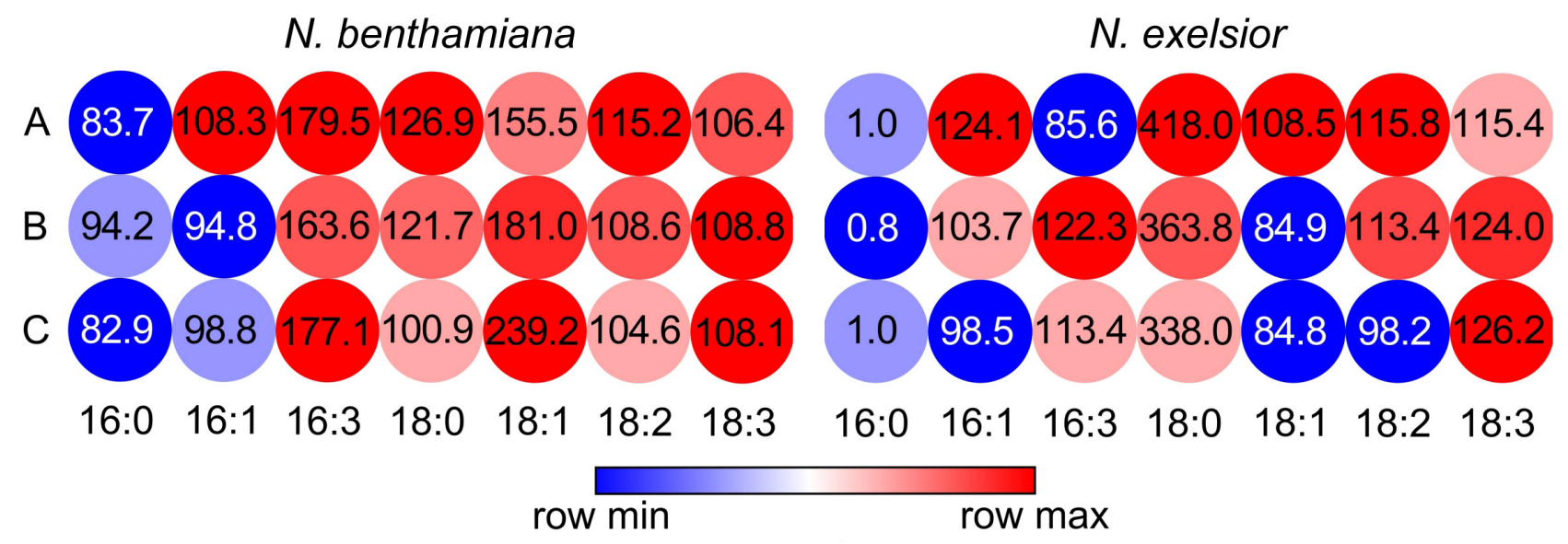

Fig. 3. A heat map of changes in the content of major fatty acidss of Nicotiana benthamiana and N. excelsior in percentage of those in the control plants (pVIG-E expression) $7 \mathrm{~d}$ after transformation. $\Delta 9$ desaturase localization: $A$ - cytosol; $B$ - plastid; $C$ - endoplasmic reticulum.

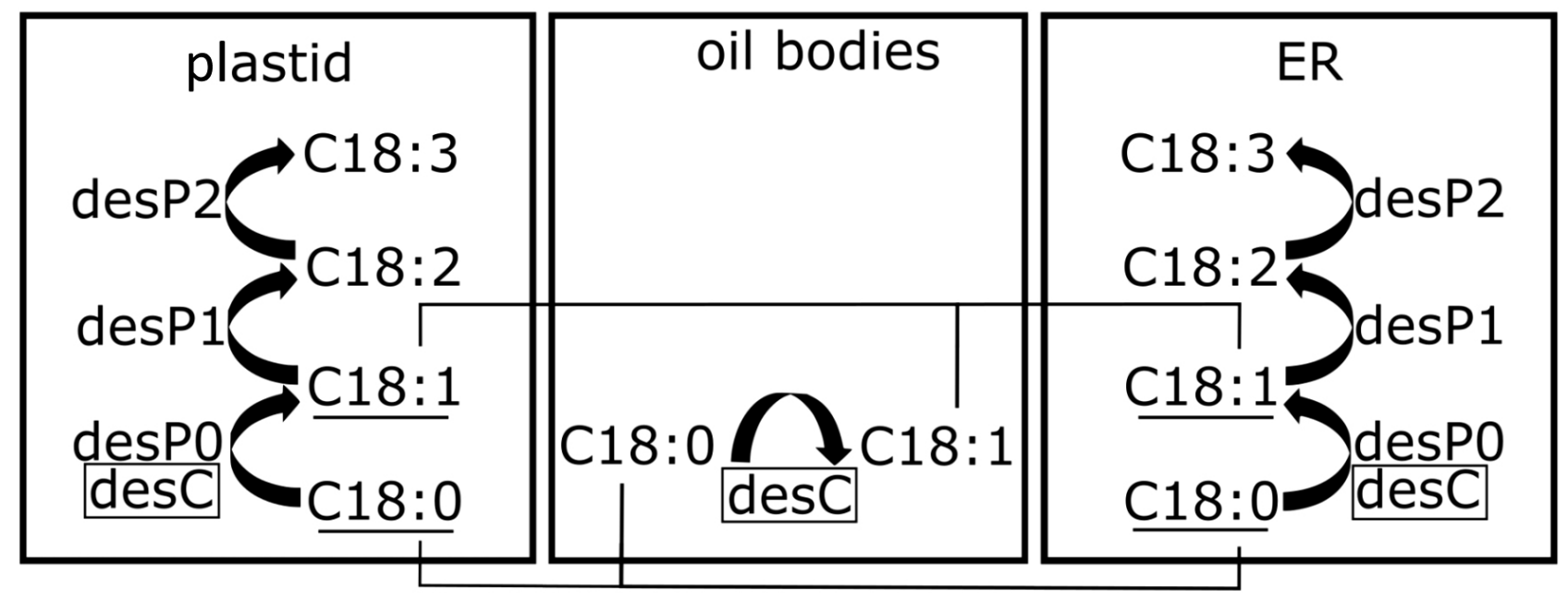

Fig. 4. The putative mechanism of functioning the heterologous $\Delta 9$-desaturase in different compartments of a plant cell. PL - plastid; ER - endoplasmic reticulum; OB - oil body; desC - $\Delta$ 9-desaturase; desP0,P1,P2 - plant desaturases $(\Delta 9, \Delta 12, \Delta 15) ; \mathrm{C} 18: 0, \mathrm{C} 18: 1, \mathrm{C} 18: 2$, C18:3 - fatty acids.

and calculated the DBI, SDR, and PDR values in the control $N$. benthamiana and $N$. excelsior plants (hereinafter referred to as wild-type plants), as well as the $N$. benthamiana and $N$. excelsior plants that had been agroinfiltrated with the Agrobacterium strain carrying the pVIG-E expression vector with constitutive expression of the reporter egfp gene (hereinafter referred to as agroinfiltrated plants).

The FA compositions of control and agroinfiltrated $N$. benthamiana leaves were measured. After agroinfiltration, only the content of the 18:1 and 16:3 FAs and SDR were significantly decreased (Table 1 Suppl.).

Comparison of the control and agroinfiltrated $N$. excelsior leaves showed a significant decrease in the 18:1 and 16:3 FA levels and SDR, as well as a significant increase in the 16:1 FA level as a result of agroinfiltration
(Table 2 Suppl.).

In general, these results suggest that neither agroinfiltration nor a possible damaging factor (wounding) activates dramatically the expression of plant desaturases, including $\Delta 9$ desaturase, because the linoleic acid (18:2) and linolenic acid (18:3) content, as well as DBI, remained unchanged, and the C18:1 content and SDR were significantly decreased after agroinfiltration in both $N$. benthamiana and $N$. excelsior leaves (Table 1, Table 2 Suppl.).

In summary, our data suggest that transient gene expression in plants upon agroinfiltration is eligible for estimating the functional activity of $\Delta 9$ desaturases and that agroinfiltration with the strain carrying the pVIG-E vector with constitutive expression of the reporter egfp gene can be used as a justified and reliable control. 
Furthermore, we used the data on the FA profile in wild-type $N$. benthamiana and $N$. excelsior to compare these species in terms of the $\mathrm{C} 18$ to $\mathrm{C} 16$ ratio and found that they considerably differed from each other. This ratio was $3: 1$ in $N$. benthamiana and $1: 1$ in $N$. excelsior. Besides, these species differed in DBI and PDR, which were significantly higher in $N$. benthamiana than in $N$. excelsior (Table 1,2 Suppl.). Thus, the two species that we used as model plants for estimating the functional activity of the heterologous desaturase can be assigned to two different types according to the FA composition: $N$. benthamiana, C18 type; $N$. excelsior, C16 type.

Then, we estimated the functional activity of the heterologous $\Delta 9$ desaturase as dependent on its localization in the plant cell. For this purpose, we used Agrobacterium strains carrying the second series of vectors containing the heterologous $\Delta 9$ desaturase gene without a signal sequence (pVIG-D9; Fig. 1), or the gene fused with signal sequences (pVIG-Lch-D9, Fig. 1) served as a control.

We analyzed the desaturase functional activity by comparing the FA profiles. Comparison of the FA compositions, SDR, PDR, and DBI showed a significant increase only in the $\mathrm{C} 18: 2$ and $\mathrm{C} 18: 3$ content, as well as DBI, in agroinfiltrated $N$. benthamiana and $N$. excelsior compared to the control. The increase in the $\mathrm{C} 18: 3$ content and DBI did not depend on the $\Delta 9$ desaturase localization in plant compartments, whereas the increase in the C18:2 was characteristic of the $\Delta 9$ desaturase localization in the chloroplasts and ER, but not in the cytosol. Furthermore, the $\mathrm{C} 16: 1$ content remained unchanged in all agroinfiltrated variants of $N$. benthamiana and $N$. excelsior, in contrast to the $\mathrm{C} 16: 0$ and $\mathrm{C} 16: 3$ content. The C16:0 content was decreased significantly in all variants of plants except for the $N$. benthamiana variant with the plastid localization. It should be emphasized, however, that a substantial (on average, tenfold) decrease in the $\mathrm{C} 16: 0$ content was characteristic of all variants of $N$. excelsior. The C16:3 content was increased significantly in all agroinfiltrated variants of $N$. benthamiana, but not $N$. excelsior, where its significant increase was observed only in the cases of cytosolic and chloroplast localizations of $\Delta 9$ desaturase, but not in the case of ER localization. Comparison of the C18:0 and C18:1 content in all agroinfiltrated variants of $N$. benthamiana and $N$. excelsior showed several differences. Specifically, the C18:0 content was increased significantly in all agroinfiltrated $N$. benthamiana and $N$. excelsior plants except for the $N$. benthamiana with the cytosolic localization of the heterologous $\Delta 9$ desaturase. However, all variants of $N$. excelsior were characterized by a significant increase in the C18:0 content (by a factor of more than three). The $\mathrm{C} 18: 1$ content exhibited different trends in agroinfiltrated $N$. benthamiana and $N$. excelsior: it was significantly increased in all $N$. benthamiana plants, irrespective of the localization of the heterologous $\Delta 9$ desaturase, whereas no significant changes in the C18:1 content were found in any $N$. excelsior variant.

Comparison of the C16:1 to C16:0 (PDR) and C18:1 to $\mathrm{C} 18: 0$ (SDR) ratios in the agroinfiltrated variants of $N$. benthamiana and $N$. excelsior also showed drastically different trends in the two species. In $N$. benthamiana, SDR was significantly increased in all variants of heterologous $\Delta 9$ desaturase localization except for the ER localization, whereas PDR was significantly increased only in the case of the ER localization. In N. excelsior, SDR was decreased, whereas PDR was increased substantially (on average, by a factor of ten) (Tables 1,2).

Thus, our data show that the $\Delta 9$ desaturase of the thermophilic cyanobacterium transiently expressed in two species considerably alters the lipid metabolism in the leaves towards a higher unsaturation of fatty acids,

Table 1. Fatty acid (FA) composition (in percentage of the total FA content) of transformed leaves of Nicotiana benthamiana $7 \mathrm{~d}$ after transformation as dependent on the cellular localization of the heterologous $\Delta 9$ desaturase. Means \pm SEs of six experiments. Asterisks indicate significant differences $(P \leq 0.05)$ from the control (pVIG-E, constitutive expression of the reporter $e g f p$ gene).

\begin{tabular}{lllllllllll}
\hline & $16: 0$ & $16: 1$ & $16: 3$ & $18: 0$ & $18: 1$ & $18: 2$ & $18: 3$ & DBI & SDR & PDR \\
\hline Control & $22.16 \pm 1.74$ & $2.40 \pm 0.35$ & $3.27 \pm 0.68$ & $3.15 \pm 0.25$ & $0.79 \pm 0.17$ & $7.42 \pm 0.25$ & $52.74 \pm 1.54$ & $1.94 \pm 0.03$ & $0.22 \pm 0.01$ & $0.10 \pm 0.01$ \\
Cytosol & $18.36 \pm 0.9 *$ & $2.37 \pm 0.40$ & $5.79 \pm 0.65^{*}$ & $3.19 \pm 0.81$ & $1.91 \pm 0.28^{*}$ & $7.77 \pm 0.23$ & $57.00 \pm 1.12 *$ & $2.05 \pm 0.03 *$ & $0.35 \pm 0.05^{*}$ & $0.12 \pm 0.01$ \\
Plastid & $20.87 \pm 0.86$ & $2.28 \pm 0.56$ & $5.35 \pm 0.94 *$ & $3.84 \pm 0.17 *$ & $1.44 \pm 0.21 *$ & $8.07 \pm 0.13 *$ & $57.39 \pm 1.43 *$ & $2.02 \pm 0.02 *$ & $0.27 \pm 0.02 *$ & $0.11 \pm 0.02$ \\
ER & $18.54 \pm 1.26^{*}$ & $2.60 \pm 0.57$ & $5.87 \pm 0.55^{*}$ & $4.01 \pm 0.25^{*}$ & $1.24 \pm 0.12^{*}$ & $8.56 \pm 0.31 *$ & $56.10 \pm 1.16^{*}$ & $2.06 \pm 0.04 *$ & $0.22 \pm 0.04$ & $0.13 \pm 0.01 *$ \\
\hline
\end{tabular}

Table 2. Fatty acid (FA) composition (in percentage of the total FA content) of transformed leaves of Nicotiana excelsior $7 \mathrm{~d}$ after transformation as dependent on the cellular localization of the heterologous $\Delta 9$ desaturase. Means \pm SEs of six experiments. Asterisks indicate significant differences $(P \leq 0.05)$ from the control (pVIG-E, constitutive expression of the reporter egfp gene).

\begin{tabular}{lllllllllll}
\hline & $16: 0$ & $16: 1$ & $16: 3$ & $18: 0$ & $18: 1$ & $18: 2$ & $18: 3$ & DBI & SDR & PDR \\
\hline Control & $38.73 \pm 1.53$ & $3.54 \pm 0.49$ & $3.84 \pm 0.17$ & $10.2 \pm 0.57$ & $1.40 \pm 0.20$ & $14.4 \pm 0.57$ & $23.61 \pm 0.88$ & $1.19 \pm 0.05$ & $0.12 \pm 0.02$ & $0.08 \pm 0.02$ \\
Cytosol & $0.40 \pm 0.18^{*}$ & $3.48 \pm 0.41$ & $4.35 \pm 0.12 *$ & $34.6 \pm 1.32 *$ & $1.19 \pm 0.18$ & $14.1 \pm 0.63$ & $29.80 \pm 0.87 *$ & $1.40 \pm 0.04 *$ & $0.03 \pm 0.02 *$ & $0.90 \pm 0.03 *$ \\
Plastid & $0.29 \pm 0.05^{*}$ & $3.67 \pm 0.80$ & $4.70 \pm 0.27 *$ & $37.2 \pm 1.33^{*}$ & $1.19 \pm 0.28$ & $16.3 \pm 0.55^{*}$ & $29.28 \pm 1.90^{*}$ & $1.33 \pm 0.04 *$ & $0.03 \pm 0.01 *$ & $0.92 \pm 0.05^{*}$ \\
ER & $0.38 \pm 0.07^{*}$ & $4.39 \pm 1.66$ & $3.28 \pm 0.66$ & $42.7 \pm 3.84^{*}$ & $1.52 \pm 0.66$ & $16.6 \pm 0.70 *$ & $27.23 \pm 0.81 *$ & $1.37 \pm 0.06^{*}$ & $0.03 \pm 0.01 *$ & $0.92 \pm 0.02 *$ \\
\hline
\end{tabular}


its functional activity depending on both the model plant species ( $N$. benthamiana or $N$. excelsior) and the specific localization of $\Delta 9$ desaturase in the plant cell.

\section{Discussion}

This study was aimed at determining 1) whether transient gene expression in model plants was in principle a suitable approach to studying the functional activity of desaturases, with heterologous $\Delta 9$ desaturase used as an example; 2) whether any of the plant cell compartments studied (the cytosol, chloroplasts, or ER) was preferable in terms of the functioning of the heterologous $\Delta 9$ desaturase; and 3 ) whether the functional activity of the heterologous $\Delta 9$ desaturase depended on the model plant species ( N. benthamiana or $N$. excelsior).

We used the $\Delta 9$ desaturase of the thermophilic cyanobacterum $S$. vulcanus because a) cyanobacterial $\Delta 9$ acyl-lipid desaturases are known to be homologous to plant acyl-CoA desaturases (Chen and Thelen 2013, Los et al. 2013, Smith et al. 2013, Bryant et al. 2016); b) thermophilic $\Delta 9$ acyl-lipid desaturase constitutively expressed in transgenic $N$. tabacum and localized in the cytosol (Orlova et al. 2003) or chloroplasts (Gerasymenko et al. 2015) of leaf cells efficiently converts stearic acid $(18: 0)$ to oleic acid (18:1), causing a considerable increase in the degree of FA unsaturation in cell membrane lipids (Orlova et al. 2003, Gerasymenko et al. 2015); and c) thermophilic $\Delta 9$ acyl-lipid desaturase recognizes and desaturates two substrates, the 16:0 and 18:0 lipid-bound FAs (palmitic and stearic acids, respectively) (Kiseleva et al. 2000), with stearic acid being a preferable substrate and the specificity towards palmitic acid being $1 \%$ of that towards stearic acid, as shown in experiments on $\Delta 9$ acyllipid desaturase expression in $N$. tabacum (Orlova et al. 2003, Gerasymenko et al. 2015).

Various gene engineering approaches, such as obtaining transgenic plants with an expression of target genes and silencing target genes using RNA interference, are used for studying the physiological roles of target genes and estimating the functional activities of their products. It should be noted that the systems of stable transformation of many plants are difficult to develop, and strategies based on ectopic expression, though applicable, are expensive and time-consuming even in model $A$. thaliana plants. On the other hand, highly efficient systems of transient expression have been developed for a number of Nicotiana species, including $N$. benthamiana and $N$. excelsior (Gerasymenko et al. 2019), and the functions of many plant proteins have been identified and clarified using the data on transient expression of target genes in these plants (Anwar et al. 2018, Ji et al. 2018; Sheludko et al. 2018, Thodberg et al. 2018, Li et al. 2019).

Plants have many FA desaturases, which are responsible for the synthesis of most PUFAs. Their important role in the variation of the FA composition of oilseeds, as well as in the vital activity of plants exposed to biotic and abiotic stress factors, has been confirmed experimentally (Orlova et al. 2003, Craig et al. 2008, Los et al. 2013, Smith et al.
2013, Zhang et al. 2014, Shi et al. 2018).

However, many representatives of this class of enzymes remain poorly studied in terms of functional activity, substrate specificity, and physiological role in plant cells. This is largely because the gene engineering approaches and manipulations used in these studies are labor- and time-consuming. Therefore, the question arises of whether the method of transient gene expression in plants, as a reliable and simple approach to studying the functions of gene products, can be used to estimate the functional activity of a heterologous desaturase.

Our data that the FA profile of $N$. benthamiana and $N$. excelsior leaves agroinfiltrated with pVIG-E remains about the same as that of wild-type plants (Tables 1, 2 Suppl.) led us to the conclusion that the method of transient gene expression in plants could be tested in experiments on the estimation of the heterologous $\Delta 9$ desaturase activity. Plants agroinfiltrated with a strain carrying the pVIG-E vector were concluded to be an appropriate control in these studies.

As noted above, thermophilic $\Delta 9$ acyl-lipid desaturase catalyzes the desaturation of two substrates, the 16:0 and 18:0 FAs (Kiseleva et al. 2000). The results of comparison of the FA profiles of wild-type $N$. benthamiana and $N$. excelsior gave grounds to assign these model plants to two different FA-composition types: $N$. benthamiana is a representative of the $\mathrm{C} 18$ type, and $N$. excelsior, of the $\mathrm{C} 16$ type. In this connection, the heterologous $\Delta 9$ desaturase in these model plants could be expected to have a preference for different substrates: the 16:0 FA in the case of $N$. excelsior and the 18:0 FA in the case of $N$. benthamiana.

In order to determine whether transient expression in model plants could be used to study the functional activity and/or substrate specificity of the heterologous $\Delta 9$ desaturase, we compared the FA compositions in the plants agroinfiltrated with the strains carrying the pVIG-E vector and with those carrying the target gene of the cyanobacterial $\Delta 9$ desaturase (referred to here as DesC plants) (Tables 1,2). Detailed comparative analysis showed that the heterologous enzyme had different effects on the FA content and composition in different Nicotiana species. Specifically, the content of one of the major saturated FAs, 16:0, in $N$. benthamiana was decreased, whereas the content of another one, 18:0, was increased. The amount of the 16:1 monounsaturated FA remained unchanged in these plants, and the amount of the 18:1 FA was increased (Table 1, Fig. 3). In addition, the DesC plants displayed considerably increased content of major trienoic FAs, 16:3 and 18:3, as well as DBI (Table 1, Fig. 3). Therefore, transient expression of the heterologous $\Delta 9$ desaturase is likely to have resulted in accumulation of end products because of higher availability of intermediate products and/or direct activation of the enzymes responsible for the subsequent stages of desaturation. This phenomenon was earlier observed in transgenic plants with stable expression of heterologous $\Delta 9$ desaturases, including that of S. vulcanus (Orlova et al. 2003, Craig et al. 2008, Gerasymenko et al. 2015, Zhao et al. 2015, Bryant et al. 2016, Dar et al. 2017).

Analysis of the FA profile in $N$. excelsior showed that 
transient expression of the heterologous $\Delta 9$ desaturase changed the amounts of major saturated FAs, the pattern of the changes differing from that in $N$. benthamiana (Fig. 3 ). The content of the 16:0 and 18:0 FAs was dramatically decreased and increased, respectively; however, whereas the amount of the 16:1 monounsaturated FA was significantly increased, that of the 18:1 FA remained at about the control level (Table 2, Fig. 3). In addition, overexpression of the heterologous $\Delta 9$ desaturase in $N$. excelsior was accompanied by a considerable change in the $\mathrm{C} 18$ to $\mathrm{C} 16$ ratio (1:3 in the wild-type and pVIG-E plants and 10:1 in the DesC plants). The increase in the $\mathrm{C} 18$ to $\mathrm{C} 16$ ratio, as well as the increase in the absolute amount of C18 FAs and the corresponding decrease in that of $\mathrm{C} 16$ FAs in the leaves of DesC plants may have resulted from an enhanced fatty acid synthase II activity, which was probably caused by an increased stearic acid utilization due to the high $\Delta 9$ desaturase expression rate (Orlova et al. 2003). Apparently, this regulatory mechanism is necessary for the homeostasis of the general level of unsaturation, which ensures the optimal fluidity of the plant cell membrane under normal environmental conditions (Los et al. 2013). Note that the content of other related unsaturated FAs, including the 16:3 and 18:3 ones, as well as DBI, were somewhat increased in the leaves of $N$. excelsior with overexpression of the heterologous $\Delta 9$ desaturase (Table 2, Fig. 3). These data indicate that the heterologous $\Delta 9$ desaturase, being responsible for the synthesis of oleic and palmitoleic acids, also strongly promotes PUFA accumulation.

Our data also demonstrate that, in general, the $S$. vulcanus $\Delta 9$ desaturase transiently expressed in plants exhibits substrate specificity for both 16:0 and 18:0 FAs in both $N$. benthamiana and $N$. excelsior (Tables 1, 2; Fig. 3), although it was earlier demonstrated that, under the conditions of stable expression in transgenic $N$. tabacum plants, this desaturase displayed preference for the 18:0 FA (Orlova et al. 2003, Gerasymenko et al. 2015).

Thus, taken together, the results of this study convincingly demonstrate that the functional activities and substrate specificities of heterologous $\Delta 9$ desaturases can be studied in detail using the model of transient expression of the target desaturase genes in $N$. benthamiana and $N$. excelsior plants.

As noted above, heterologous desaturases catalyzing the 18:0 to $18: 1$ conversion effectively function in plants in the cases of both cytosolic (Orlova et al. 2003, Zhao et al. 2015) and plastid (Craig et al. 2008, Gerasymenko et al. 2015) localizations, which eventually leads to the formation of PUFAs due to the activities of specific desaturases localized in the ER and plastids.

Here, we also attempted at determining whether different plant cell compartments (the cytosol, chloroplasts, and ER) had preferences for the functioning of the heterologous $\Delta 9$ desaturase, mainly in terms of the PUFA content and DBI. For this purpose, we determined the FA composition of $N$. benthamiana and $N$. excelsior leaves overexpressing the cyanobacterial des $C$ gene with different localizations of the DesC protein. We used well-characterized signal sequences, namely, 1) the
N-terminal transit peptide of the RuBisCO small subunit, which ensures the localization of target proteins in the chloroplasts (Vyacheslavova et al. 2012, Tyurin et al. 2017); 2) the N-terminal transit peptide of legumin B4 (LeB4) of Vicia faba; and 3) the ER retranslation signal SRKDEL introduced into the C-terminal region of the target protein to ensure its transport to the ER and its retention there (Alanen et al. 2011, Vyacheslavova et al. 2012, Tyurin et al. 2017). We used the method of obtaining protoplasts from a plant tissue agroinfiltrated with bacterial strains carrying different expression vectors, which we developed and tested earlier, and fluorescence microscopy to demonstrate that the signal sequences ensured the localization of the heterologous $\Delta 9$ desaturase in the target subcellular compartments (Fig. 2).

Subsequent comparison of the FA composition of agroinfiltrated DesC plants with different localizations of the heterologous $\Delta 9$ desaturase and that of control plants (pVIG-E) showed that, in general, the $S$. vulcanus $\Delta 9$ desaturase transiently expressed in different plant cell compartments caused a significant increase in both the PUFA content and DBI (Tables 1, 2; Fig. 3) in the leaves of both $N$. benthamiana and $N$. excelsior. The significant increase in the PUFAs in the case of the plastid localization of the heterologous $\Delta 9$ desaturase is satisfactorily explained by the presence of stearic acid (18:0), a substrate of this enzyme, in plastids and formation of PUFAs through further desaturation of the $\Delta 9$ desaturase reaction product, oleic acid (18:1), by specific plant desaturases also localized in this compartment (Dar et al. 2017) (Fig.4).

In the ER, the heterologous enzyme desaturates the 18:0 FA transported as part of the FA-CoA pool from the cytosol (usually the oil body). The resultant 18:1 FA is also converted into PUFAs by specific plant desaturases localized in the ER (Los et al. 2013, Dar et al. 2017) (Fig. 4).

Regarding the localization and functioning of $\Delta 9$ desaturase in the cytosol, the following mechanism of the increase in the PUFA content can be supposed. Probably, the mechanism of FA exchange between the plastid and ER membranes is employed in this case; i.e., the heterologous enzyme desaturates the 18:0 FA contained in the cytosolic FA-CoA pool, and the desaturation product, the 18:1 FA, is transported to the plastids and/or ER, where PUFA formation is catalyzed by specific desaturases contained in these compartments (Fig. 4).

The results of this study allow us to recommend both $N$. benthamiana and $N$. excelsior as model plants for estimating the functional activities and/or substrate specificities of not only the heterologous $\Delta 9$ desaturase studied here but also the desaturases that convert the 18:1 FA into PUFAs. Since the model plants used here, $N$. benthamiana and $N$. excelsior, are characterized by substantial differences in FA composition, including the $\mathrm{C} 18$ to $\mathrm{C} 16$ ratio, the choice of the model plant will certainly depend on the purpose of each particular study.

A specific advantage of the expression vectors for transient expression of target genes in plants that we engineered earlier (Vyacheslavova et al. 2012) should be specially noted. It is determined by an additional 
expression cassette for the gene of the p19 tomato virus protein, a suppressor of posttranscriptional gene silencing controlled by the constitutive TCTP promoter, incorporated in the vectors. This makes it possible a) to overcome the mechanism of posttranscriptional silencing upon overexpression of heterologous genes in plants, which is usually triggered in the case of transient expression and interferes with effective expression of the heterologous gene, and $b$ ) to use a single bacterial strain for agroinfiltration, in contrast to the routine procedure of cotransformation with two Agrobacterium strains carrying different vectors, one with the p19 protein and the other with the target gene (Vyacheslavova et al. 2012, Tyurin et al. 2017).

Thus, we have proposed and tested a model of transient gene expression in plants that have been found applicable to the study of the functional activities and substrate specificities of heterologous desaturases. Transient expression is a simpler, less time-consuming, and less expensive approach than obtaining transgenic plants for both stable gene overexpression and gene silencing. The use of transient expression, in particular, for estimating the localization, functional activity, and substrate specificity of desaturases of different origins makes it possible to maintain molecular reproduction of new genotypes containing better combinations of target alleles and, combined, e.g., with genome-editing technologies, lay the basis for a new generation of transgenes, which has important implications at the global level.

\section{References}

Alanen, H.I., Raykhel, I.B., Luukas, M.J., Salo, K.E., Ruddock L.W.: Beyond KDEL: the role of positions 5 and 6 in determining ER localization. - J. mol. Biol. 409: 291-297, 2011.

Anwar, M., Wang, G., Wu, J, Waheed, S., Allan, A.C., Zeng, L.: Ectopic overexpression of a novel R2R3-MYB, NtMYB2 from Chinese Narcissus represses anthocyanin biosynthesis in tobacco. - Molecules 23: 781, 2018.

Berestovoy, M.A., Tyurin, A.A., Kabardaeva, K.V., Sidorchuk, Y.V., Fomenkov, A.A., Nosov, A.V., Goldenkova-Pavlova, I.V.: Transient gene expression for the characteristic signal sequences and the estimation of the localization of target protein in plant cell. - Bio-protocol. 8: e2738, 2018.

Bryant, F.M., Munoz-Azcarate, O., Kelly, A.A., Beaudoin, F., Kurup, S., Eastmond, P.J.: Acyl-acyl carrier protein desaturase 2 and 3 are responsible for making omega-7 fatty acids in the Arabidopsis aleurone. - Plant Physiol. 172: 154-162, 2016.

Chen, M., Thelen, J.J.: Acyl-lipid desaturase 2 is required for chilling and freezing tolerance in Arabidopsis. - Plant Cell 25: 1430-1444, 2013.

Craig, W., Lenzi, P., Scotti, N., De Palma, M., Saggese, P., Carbone, V., McGrath Curran, N., Magee, A.M., Medgyesy, P., Kavanagh, T.A., Dix, P.J., Grillo, S., Cardi, T.: Transplastomic tobacco plants expressing a fatty acid desaturase gene exhibit altered fatty acid profiles and improved cold tolerance. Transgenic Res. 17: 769-872, 2008.

Dar, A.A., Choudhury, A.R, Kancharla, P.K., Arumugam, N.: The FAD2 gene in plants: occurrence, regulation, and role. - Front. Plant. Sci. 8: 1789, 2017.
Gerasymenko, I.M., Sakhno, L.A., Kyrpa, T.N, Ostapchuk, A.M., Hadjiev, T.A., Goldenkova-Pavlova, I.V, Sheludko, Y.V.: Characterization of Nicotiana tabacum plants expressing hybrid genes of cyanobacterial $\Delta 9$ or $\Delta 12$ acyllipid desaturases and thermostable lichenase. - Russ. J. Plant Physiol. 62: 283-291, 2015.

Gerasymenko, I., Sheludko, Y., Fräbel, S., Staniek, A., Warzecha, H.: Combinatorial biosynthesis of small molecules in plants: engineering strategies and tools. - Methods Enzymol. 617: 413-442, 2019.

Ji, X.J., Mao, X., Hao, Q.T., Liu, B.L., Xue, J.A., Li, R.Z.: Splice variants of the castor WRI1 gene upregulate fatty acid and oil biosynthesis when expressed in tobacco leaves. - Int. J. mol. Sci. 19: 146, 2018.

Kiseleva, L.L., Serebriiskaya, T.S., Horváth, I., Vigh, L., Lyukevich, A.A., Los, D.A.: Expression of the gene for the delta9 acyl-lipid desaturase in the thermophilic cyanobacterium. - J. mol. Microbiol. Biotechnol. 2: 331-338, 2000.

Li, S., Zhao, J., Zhai, Y., Yuan, Q., Zhang, H., Wu, X., Lu, Y., Peng, J., Sun, Z., Lin, L., Zheng, H., Chen, J., Yan, F.: The hypersensitive induced reaction 3 (HIR 3) gene contributes to plant basal resistance via an EDS 1 and salicylic aciddependent pathway. - Plant J. 98: 783-797, 2019.

López Alonso, D., García-Maroto, F., Rodríguez-Ruiz, J., Garrido, J.A., Vilches, M.A.: Evolution of the membranebound fatty acid desaturases. - Biochem. syst. Ecol. 31: 1111$1124,2003$.

Los, D.A., Mironov, K.S., Allakhverdiev, S.I.: Regulatory role of membrane fluidity in gene expression and physiological functions. - Photosynth. Res. 116: 489-509, 2013.

Maali, R, Shimshilashvili, Kh.R., Pchelkin, V.P., Tsydendambaev, V.D., Nosov, A.M., Los, D.A., Goldenkova-Pavlova, I.V.: Comparative expression in Escherichia coli of the native and hybrid genes for acyl-lipid delta(9) desaturase. - Genetika 43: 176-182, 2007.

Napier, J.A.: The production of unusual fatty acids in transgenic plants. - Annu. Rev. Plant. Biol. 58: 295-319, 2007.

Orlova, I.V., Serebriiskaya, T.S., Popov, V., Merkulova, N., Nosov, A.M., Trunova, T.I., Tsydendambaev, V.D., Los, D.A.: Transformation of tobacco with a gene for the thermophilic acyl-lipid desaturase enhances the chilling tolerance of plants. - Plant Cell Physiol. 44: 447-450, 2003.

Peng, D, Zhou, B, Jiang, Y., Tan, X., Yuan, D., Zhang, L.: Enhancing freezing tolerance of Brassica napus L. by overexpression of a stearoyl-acyl carrier protein desaturase gene $(S A D)$ from Sapium sebiferum (L.) Roxb. - Plant Sci. 272: 32-41, 2018.

Piruzian, E.S, Goldenkova, I.V., Musiychuk K.A., Kobets, N.S., Arman, I.P., Bobrysheva, I.V., Chekhuta, I.A., Glazkova D.: A reporter system for prokaryotic and eukaryotic cells based on the thermostable lichenase from Clostridium thermocellum. Mol. Genet. Genomics 266: 778-786, 2002.

Sheludko, Y.V., Gerasymenko I.M., Warzecha H.: Transient expression of human cytochrome P450s 2D6 and 3A4 in Nicotiana benthamiana provides a possibility for rapid substrate testing and production of novel compounds. Biotechnol. J. 13: 1700696, 2018.

Shi, Y., Yue, X., An, L.: Integrated regulation triggered by a cryophyte $\omega-3$ desaturase gene confers multiple-stress tolerance in tobacco. - J. exp. Bot. 69: 2131-2148, 2018.

Sidorov, R.A., Zhukov, A.V., Vereshchagin, A.G., Tsydendambaev, V.D.: Occurrence of fatty acid lower-alkyl esters in euonymus fruits. - Russ. J. Plant Physiol. 59: 326332, 2012.

Smith, M.A., Dauk, M., Ramadan, H., Yang, H., Seamons, L.E., 
Haslam, R.P., Beaudoin, F., Ramirez-Erosa, I., Forseille, L.: Involvement of Arabidopsis acyl-coenzyme A desaturaselike 2 (At2g31360) in the biosynthesis of the very-long-chain monounsaturated fatty acid components of membrane lipids. - Plant Physiol. 161: 81-96, 2013.

Thodberg, S., Del Cueto, J., Mazzeo, R., Pavan, S., Lotti, C., Dicenta, F., Jakobsen Neilson, E.H., Møller, B.L., SánchezPérez, R.: Elucidation of the amygdalin pathway reveals the metabolic basis of bitter and sweet almonds (Prunus dulcis). - Plant Physiol. 178: 1096-1111, 2018.

Troncoso-Ponce, M.A., Barthole, G., Tremblais, G., To, A., Miquel, M., Lepiniec, L., Baud, S.: Transcriptional activation of two delta-9 palmitoyl-ACP desaturase genes by MYB115 and MYB118 is critical for biosynthesis of omega-7 monounsaturated fatty acids in the endosperm of Arabidopsis seeds. - Plant Cell 28: 2666-2682, 2016.

Tyurin, A.A., Kabardaeva, K.V., Berestovoy, M.A., Sidorchuk, Yu.V., Fomenkov, A.A., Nosov, A.V., Goldenkova-Pavlova, I.V.: Simple and reliable system for transient gene expression for the characteristic signal sequences and the estimation of the localization of target protein in plant cell. - Russ. J. Plant Physiol. 64: 672-679, 2017.

Vyacheslavova, A.O., Mustafaev, O.N., Tyrin, A.A., Shimshilashvili, K.R., Berdichevets, I.N., Shayakhmetova, D.M., Goldenkov, M.A., Fadeev, V.S., Sheludko, Yu.V., Goldenkova-Pavlova, I.V.: Set of module vectors for stable or transient expression of heterologous genes in plants. -
Genetika 48: 1046-1056, 2012.

Wang, H.S., Chao Yu, C., Tang, X.F., Wang, L.Y., Dong, X.C., Meng, Q.W.: Antisense-mediated depletion of tomato endoplasmic reticulum omega-3 fatty acid desaturase enhances thermal tolerance. - J. integr. Plant. Biol. 52: 568$577,2010$.

Wang, J.J, Liu, H.R, Gao, J., Huang, Y.J., Zhang, B., Chen, K.S.: Two $\omega-3$ FADs are associated with peach fruit volatile formation. - Int. J. mol. Sci. 17: 464, 2016.

Xue, Y., Chen, B., Win, A.N., Chun Fu, Lian, J., Liu, X., Wang, R., Zhang, X., Chai, Y.: Omega-3 fatty acid desaturase gene family from two $\omega-3$ sources, Salvia hispanica and Perilla frutescens: cloning, characterization and expression. - PLoS ONE 13: e0191432, 2018.

Zhang, J., Li, J., Garcia-Ruiz, H., Bates, P.D., Mirkov, T.E., Wang, $\mathrm{X}$. A stearoyl-acyl carrier protein desaturase, NbSACPD-C, is critical for ovule development in Nicotiana benthamiana. Plant J. 80: 489-502, 2014.

Zhang, Y., Maximova, S.N., Guiltinan, M.J.: Characterization of a stearoyl-acyl carrier protein desaturase gene family from chocolate tree, Theobroma cacao L. - Front. Plant. Sci. 6: 239, 2015.

Zhao, N., Zhang, Y., Li, Q., Li, R., Xia, X., Qin, X., Guo, H. Identification and expression of a stearoyl-ACP desaturase gene responsible for oleic acid accumulation in Xanthoceras sorbifolia seeds. - Plant Physiol. Biochem. 87: 9-16, 2015. 